\title{
Design of a Novel Test Fixture to Measure Rotational Core Losses in Machine Laminations
}

\author{
Natheer Alatawneh, Student Member, IEEE, and Pragasen Pillay, FIEEE
}

\begin{abstract}
The need for testing magnetic materials used in electric machine laminations and measurements under a rotating field is of importance in machine design. In order to obtain satisfactory experimental data, the design of the measurement apparatus deserves particular attention. The purpose of this paper is to propose a novel design of a magnetic circuit based on the Halbach array, which generates a uniform flux density inside the test specimen for the measurement of rotational core loss. The proposed design is simulated, prototyped, and experimental tests are performed on two different samples of M19G29 and M36G29 at three different frequencies $(60 \mathrm{~Hz}, 400 \mathrm{~Hz}$, and $1 \mathrm{kHz})$. The field-metric method is used in this work to evaluate the rotational core losses.
\end{abstract}

Keywords: Rotational core losses, Halbach array, magnetic materials, electrical steel.

\section{INTRODUCTION}

In the cores of rotating $\mathrm{AC}$ machines and 3-phase $\mathrm{T}$-joint transformers many zones are subjected to a rotating magnetic field. This loss due to the rotational flux is different from the well known pulsating loss, and it is reported by some researchers that rotational core losses in motors can amount to more than $50 \%$ of the total core losses [1]. Characterization and quantification of core losses in the stator yoke of $\mathrm{AC}$ machines shows that the rotational core loss is concentrated in the root of the teeth [2].

Different types of test fixtures and measuring techniques have been proposed and used for measurements of rotational core loss in electrical steel laminations. Technically, it is still a challenge to realize both a high and uniform magnetic flux density within a wide area of the sample under test. Because of that, importance has been placed on developing magnetizing circuits which are capable of performing 2-D rotating field tests $[3,4]$.

In this paper, a novel design of a magnetizing circuit is presented, simulated, and a prototype implemented to obtain a highly uniform flux density inside the test sample. This magnetizing circuit is based on an electromagnetic system which is used to establish a Halbach pattern; electromagnets are used instead of permanent magnets. To the author's knowledge, the electromagnetic Halbach array has not been used in core loss measuring apparatus. The originality of this work can be seen from many different angles and summarized as follow: a) Using electromagnets instead of PMs leads to a controllable Halbach array, (controllability in field magnitude and the signal shape), which is lacking in the PM array. Also, it allows for a more compact sized array by using the same core for two different field directions of electromagnets.

b) By using an electromagnetic Halbach array, a more uniform flux density is obtained than any other prior magnetizing circuit design, as proved in [5].

c) The ability to generate a uniform and homogeneous flux density inside the sample gives the flexibility to use a new technique for wrapping the $\boldsymbol{B}$ coils around the sample, previously holes had to be drilled which caused a degradation in the sample magnetic properties.

d) Wrapping the $\boldsymbol{B}$ coils into two blocks and leaving the sample middle area empty, gives the opportunity for the $\boldsymbol{H}$ coils to be placed directly on the sample which gives a higher accuracy for magnetic field intensity measurements on the sample surface. In prior designs the $\boldsymbol{B}$ coils were between the sample and $\boldsymbol{H}$ coils which decreases the accuracy of the measured $\boldsymbol{H}$.

e) In this novel design, the $\boldsymbol{B}$ coil width is much higher (180 mm) when compared to the typical coil width (20 $\mathrm{mm}$ ) which decreases the possibility of misalignments in $\boldsymbol{B}$ coils during the process of threading the coils. This improves loss measurements in the clockwise and counterclockwise directions.

Several researchers have studied rotational core losses, so the test equipment and measurement techniques have not been standardized yet, which yields high discrepancy in loss prediction [6]. Because of that more attention should be paid to work in this research area. Reliable data for the industry is still a challenge. This new design test fixture offers rotational core loss data which can be used in the comparison with other data obtained from different laboratories to achieve agreed standardization. The general behavior of the rotational core losses are well known, but many physical phenomena associated with measurements are still vague and unsolved [7], such as the difference in loss in $\mathrm{CW}$ and $\mathrm{CCW}$ rotating field, the negative power which has appeared in one loss component measurement, and the effect of harmonics and its relation with the B-H loop trajectory.

This paper is organized as follows: Section II explains the magnetizing circuit design considerations. The electromagnetic Halbach array concept is discussed in 
section III. Section IV describes the test fixture structure. Section $\mathrm{V}$ presents the measurement set-up. The experimental results and conclusion are presented and discussed in sections VI and VII respectively.

\section{Magnetizing Circuit Design CONSIDERATIOnS}

\section{A. Halbach array principle}

In general, the Halbach array is an arrangement of permanent magnets in a special orientation that have the ability to create a one-sided flux of magnetization while canceling it on the other side [8,9]. In the circular format, the magnets are arranged in predetermined angles in a circular frame where the flux is confined entirely within the frame [10]. As an example, fig. 1 (a) shows an 8-pole Halbach circular design where 8 permanent magnets are arranged in specific angles, i.e. arrows show the flux directions. The total magnetic flux is confined within the frame and is directed entirely in one direction in the air gap at the center. Fig. 1 (b) shows the simulation of this format arrangement. The magnets used in this simulation are cylindrical in shape.

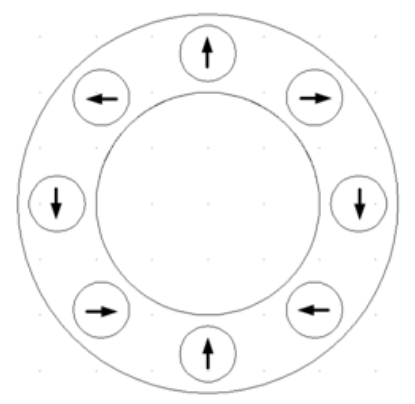

(a)

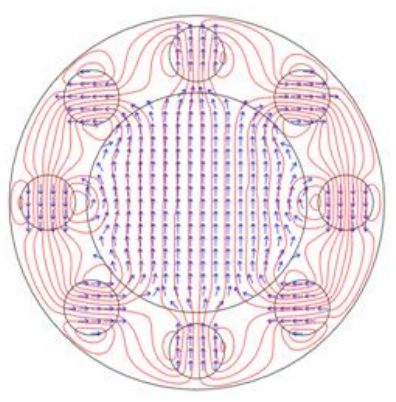

(b)
Fig. 1: Circular Halbach design of 8 cylindrical magnets which produces one sided flux in the y-axis.(a) circuit design (b) simulation result.

Of importance in the design is the determination of the deflection angles of the magnets to enhance the flux in only one direction in the air gap. The deflection angle $\delta$ can be calculated according to (1).

$$
\delta=2 \times \frac{360^{\circ}}{\text { number of poles }}
$$

\section{B. Choosing size and number of poles}

The size of poles can be determined by considering the sample dimensions, and the air gap in the fixture, as shown in fig. 2. Then equation (2) can be used to calculate the pole radius $r_{2}$.

$$
\frac{\sin \theta}{2 \times r_{2}}=\frac{\sin \alpha}{r_{1}+r_{2}}
$$

$r_{l} \quad$ is the sample radius plus the air gap length.

$r_{2}$ is the pole radius.

$\theta$ is the central angle whose sides pass through centers of two adjacent poles.

$\alpha \quad$ is equal to $\alpha=(\pi-\theta) / 2$.

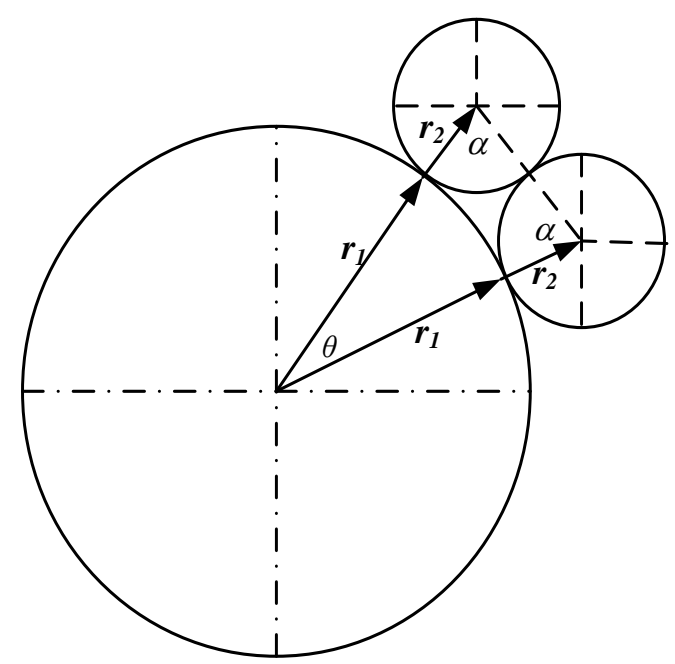

Fig. 2: Poles around the central air gap.

The number of poles in the magnetic array is an important factor which determines the flux density level and the homogeneity of the magnetic flux inside the sample. In this design, poles are arranged in a circular path to develop a one-sided flux direction in any desired direction.

Simulations are performed to examine the most suitable number of poles for a circular sample of $20 \mathrm{~cm}$ diameter, and $1 \mathrm{~cm}$ air gap. These include 8,16 , and 32 pole circuits. The geometrical shapes and dimensions (Figs. 3, 4, and 5) are designed according to the equations (1) and (2). The flux lines are shown at an angle of $45^{\circ}$.

Figs. 6, 7, and 8 show the magnetic flux density profile along the $x$-axis of the sample in the three circuits of different pole numbers. A large pole number requires more work in prototyping. Results illustrate that the flux variation within the sample in the 8-pole circuit happens not only at the edges but also in the middle of the sample, with a standard deviation of 0.0247 . In the 16-pole design the variation is limited and appears at edges, with a standard deviation of 0.0209 , where the flux density distribution is considered to be more homogeneous.

where 


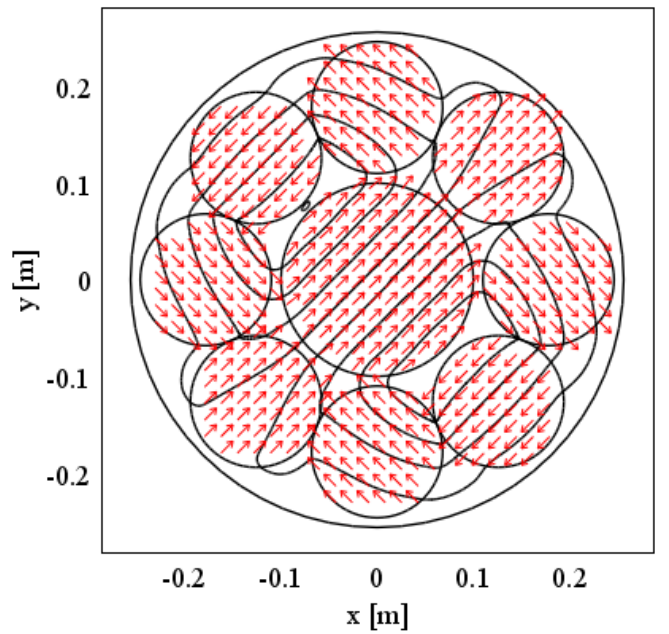

Fig. 3: Simulation showing resultant of two orthogonal equal vectors of 8 -pole Halbach array producing $45^{\circ}$ direction flux.

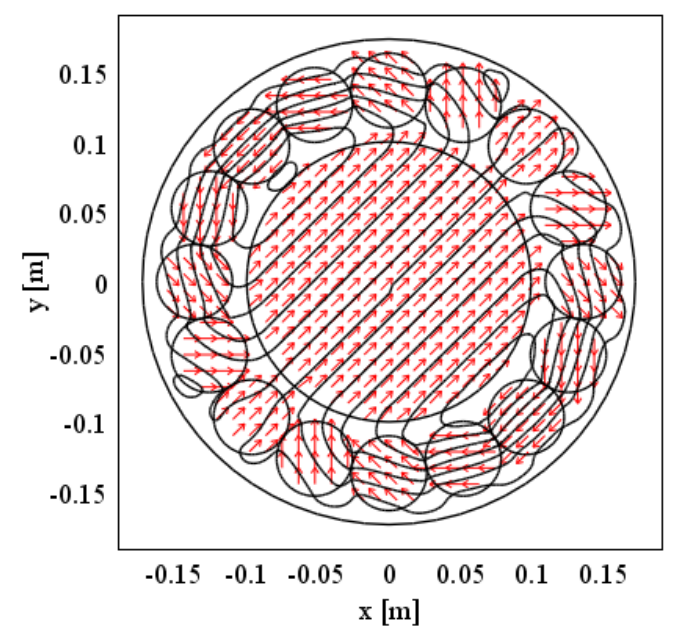

Fig. 4: Simulation showing resultant of two orthogonal equal vectors of 16-pole Halbach array producing $45^{\circ}$ direction flux.

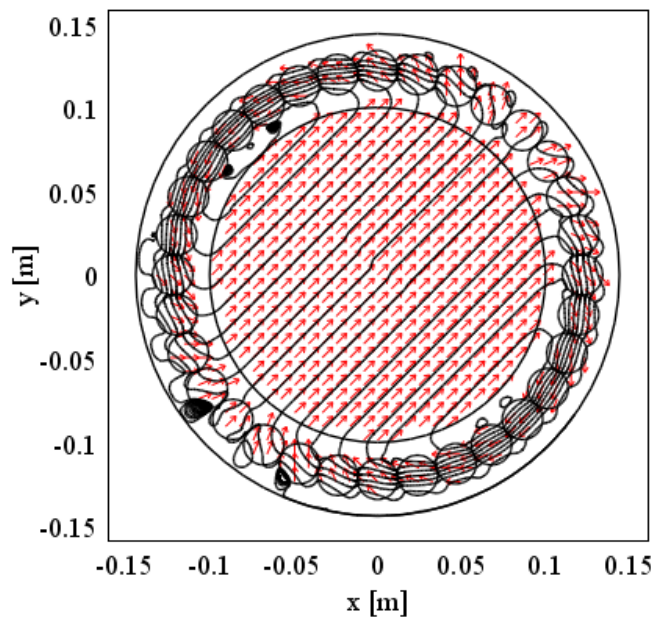

Fig. 5: Simulation showing resultant of two orthogonal equal vectors of 32-pole Halbach array producing $45^{\circ}$ direction flux

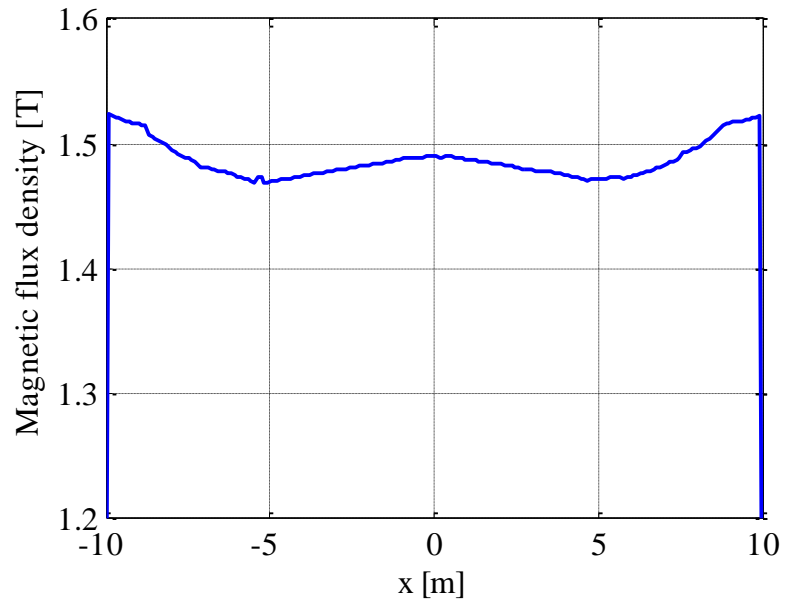

Fig. 6: The magnetic flux density profile along the $x$-axis of the sample in 8 -pole circuit.

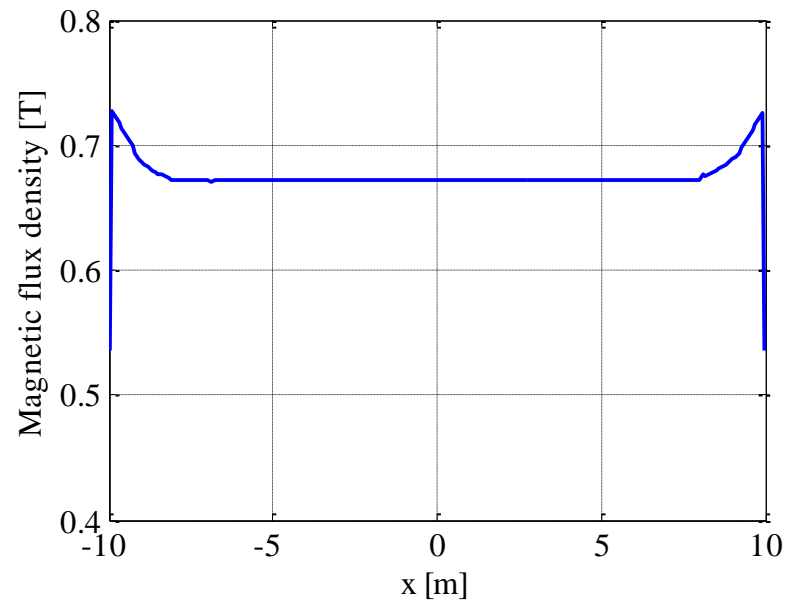

Fig. 7: The magnetic flux density profile along the $x$-axis of the sample in 16-pole circuit.

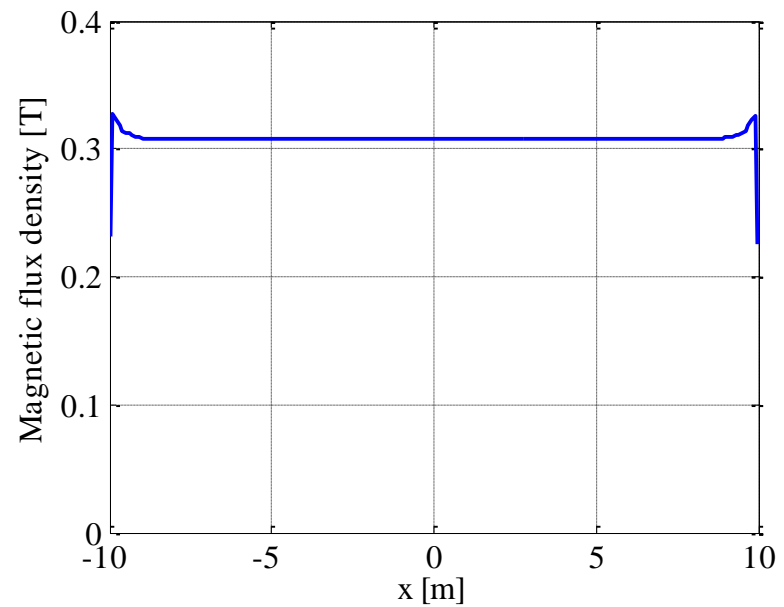

Fig. 8: The magnetic flux density profile along the $x$-axis of the sample in 32-pole circuit. 


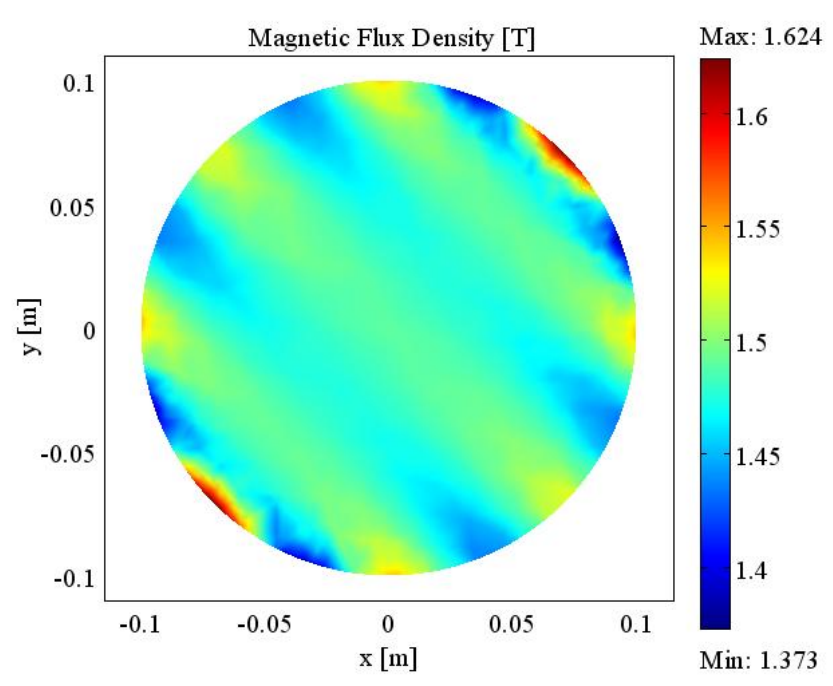

Fig. 9: The 2-D flux density distribution of the sample in an 8-pole circuit.

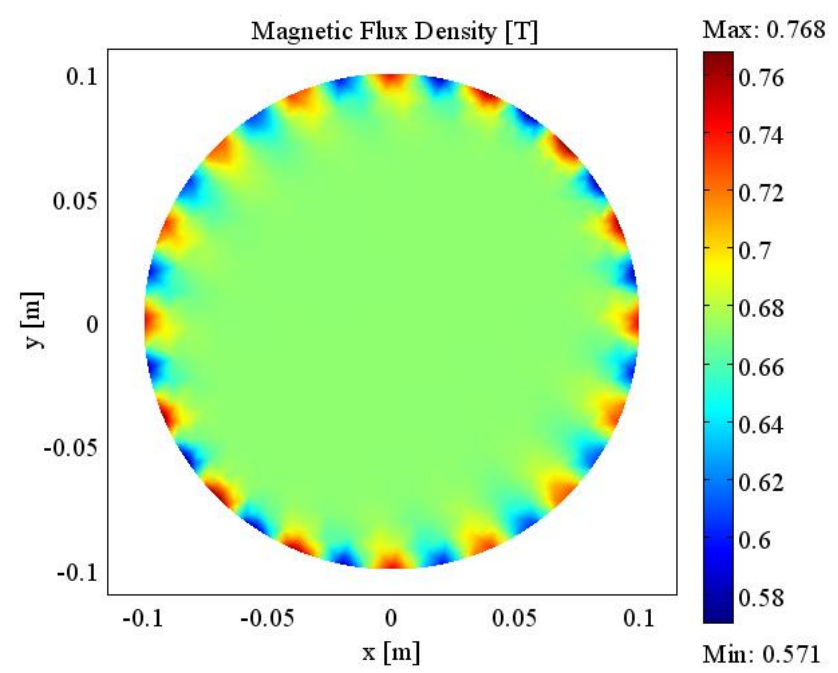

Fig. 10: The 2-D flux density distribution of the sample in a 16-pole circuit.

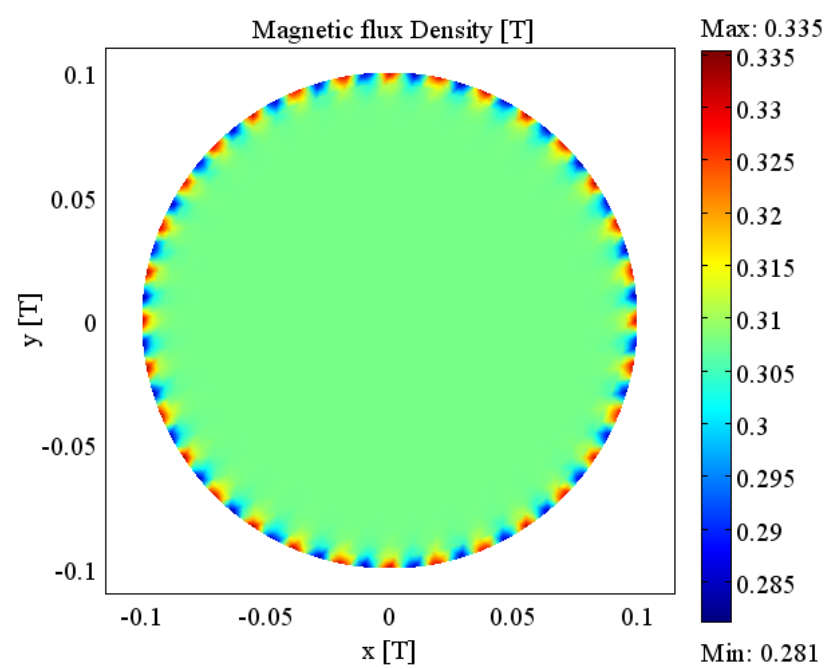

Fig. 11: The 2-D flux density distribution of the sample in a 32-pole circuit.
With the 32-pole design, the variation in the flux is the least. The results are presented in Table I. As a consequence, by increasing the number of poles, the variability of the magnetic field inside the sample decreases, and the flux density distribution becomes more homogeneous. To compromise between the prototyping effort and flux uniformity distribution, the 16-pole design was chosen. The 2-D flux density distributions of the same sample for the three different circuits are shown in figs. 9, 10, and 11.

TABLE I

StANDARD DEVIATION For FluX DENSITY Distribution In EACH SAMPLE IN THREE Circuits OF DifFERENT POLES.

\begin{tabular}{cc}
\hline \hline Number of poles & Standard Deviation \\
\hline \hline 8 poles circuit & 0.0247 \\
\hline 16 poles circuit & 0.0209 \\
\hline 32 poles circuit & 0.0059 \\
\hline
\end{tabular}

The reason for the reduction in the flux density distribution is explained from the fundamental Halbach equation. Dr. Halbach described the magnetic field inside the cylindrical array by the following equation [11]:

$$
B_{o}=B_{r} \ln \left[\frac{r_{o}}{r_{i}}\right] \frac{\sin \left[\frac{2 \pi}{m}\right]}{\left[\frac{2 \pi}{m}\right]}
$$

$B_{o}$ is the field produced inside the working aperture. $B_{r}$ is the remanence of the magnetic field of the permanent magnet material. $r_{i}$ and $r_{o}$ are the inside and outside radii of the magnet array, respectively. $m$ is number of segments used.

By using equation (3), with many poles of different dimensions designed according to (2), and a constant magnetization $(\boldsymbol{M})=1170[\mathrm{kA} / \mathrm{m}]$, the field produced inside the gap is illustrated in fig. 12. By increasing the number of poles, the pole dimensions become smaller, and the flux density inside the array decreases.

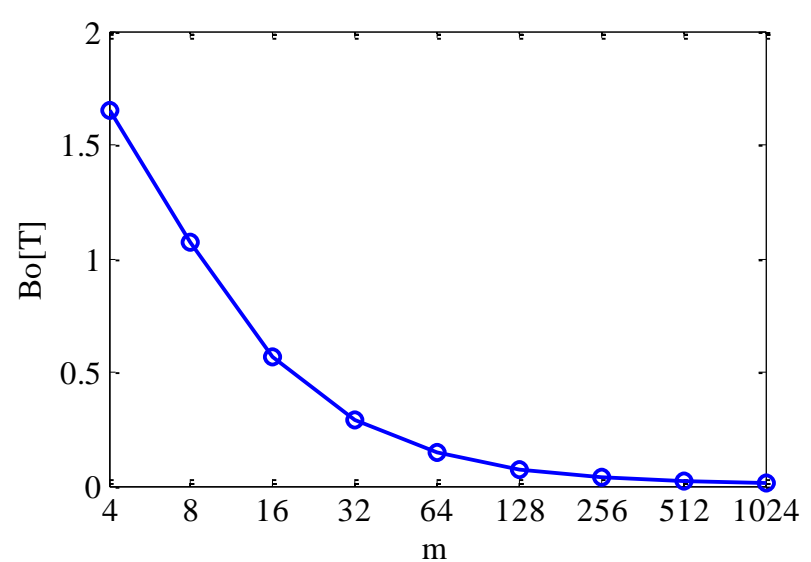

Fig. 12: The produced flux density in the gap of the Halbach array with different poles. 


\section{THE EleCtromagnETIC HALBACH ARRAY}

Recently, many industrial applications show a strong interest in the PM Halbach array because of its attractive features, where it has used effectively in the PM motor designs [12]. To date the Halbach array is known and presented using permanent magnets. In this new design, the magnetizing circuit is based on an electromagnetic system which is used to establish the Halbach pattern; electromagnets are used instead of permanent magnets, where coils are wrapped around magnetic cores in order to generate the flux. By using electromagnets, a controllable array in field magnitude and in signal shape is obtained. It also allows for a more compact sized array by using the same core for two different directions of electromagnets. This is the first application of an electromagnetic Halbach array for material testing.

Consider the 16-pole circuit, where the arrangement of the poles in the frame is in fig. 13 (a), where a deflection of angle $\delta\left(\delta=45^{\circ}\right)$ is applied on each pole with respect to the previous one. This electromagnetic Halbach array generates a flux density in the $x$-axis in the left direction. The same idea can be applied on the $y$-axis array as shown in fig. 13 (b), where the net flux in gap goes in the positive $y$-direction. If both arrays are combined by wrapping two coils on the same core (each coil is related to a different array), and both arrays are excited with the same input signals, then the result of the two perpendicular equal vectors is a resultant at $45^{\circ}$. The flux direction inside the sample can be varied in many ways: (a) Rotating the poles, which is not preferable, since it is difficult and not practical to move them mechanically. (b) Changing the amplitudes of the input signals, where the difference between the two magnitudes gives an ability to control the resultant. Fig. 14 shows the flux lines for finite element analysis (FEA) of 16-pole electromagnetic Halbach array, the resultant flux vectors in the samples at $45^{\circ}$, and $60^{\circ}$, where the flux paths through the top poles are shown in fig. 15. Fig. 16 depicts the 2-D flux density distribution in the sample of a Halbach array with a resultant net vector at $45^{\circ}$.

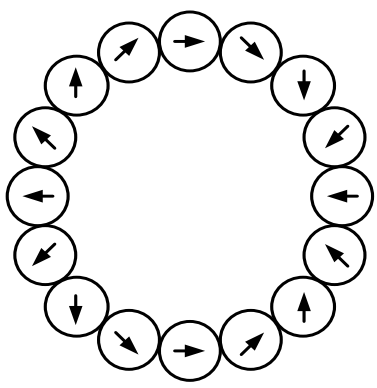

(a)

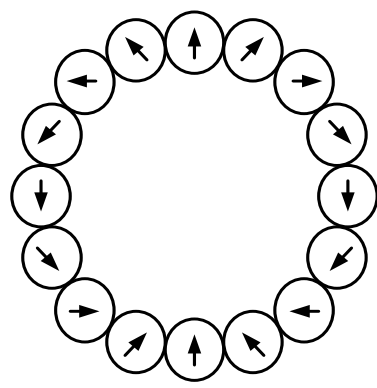

(b)
Fig. 13: The flux direction in each pole of a 16-pole Halbach array to produce a uni-directional flux. (a) in $x$-axis (b) in $y$-axis.

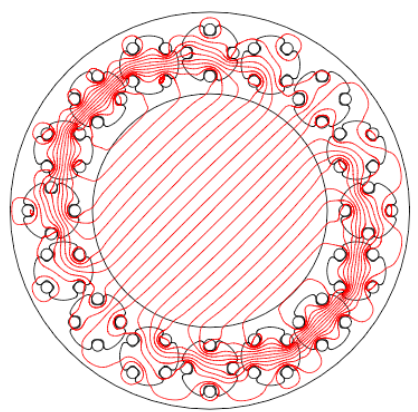

(a)

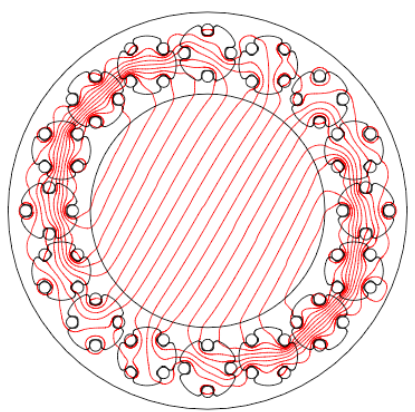

(b)
Fig. 14: Flux lines from FEA showing resultant the net vector at (a) $45^{\circ}$ (b) $60^{\circ}$

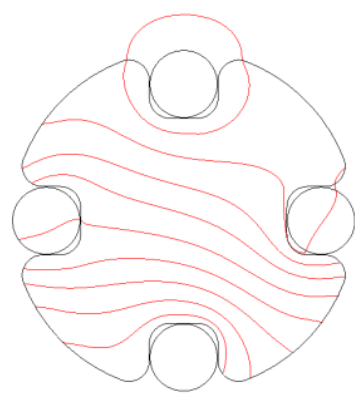

(a)

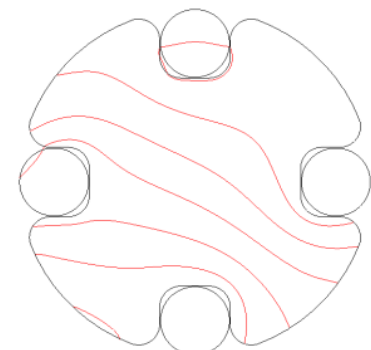

(b)
Fig. 15: Flux paths through the top pole of an electromagnetic Halbach array for a resultant vector at (a) $45^{\circ}$ (b) $60^{\circ}$.

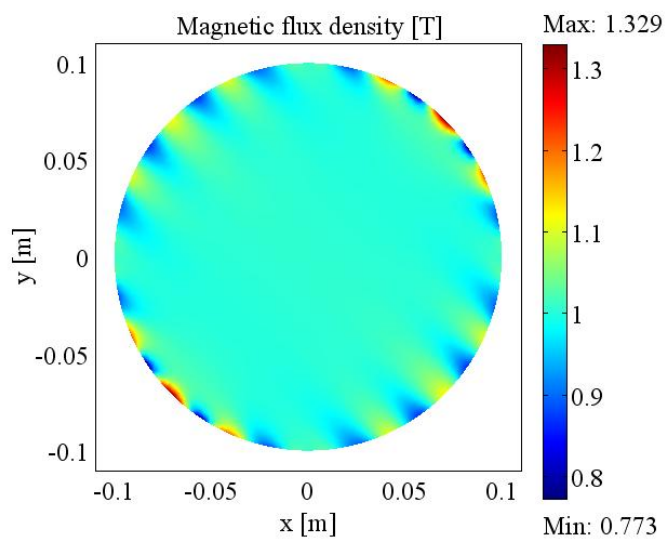

Fig. 16: The 2-D flux density distribution in the sample with Halbach array and a resultant net vector at $45^{\circ}$.

The rotating magnetic field is produced automatically by injecting both arrays with time varying voltage waveforms. The voltage waveforms are out of phase by angle $\varphi$, and orthogonal mechanically. Suppose that the arrays are injected by sinusoidal signals, then the net vector produced in the test specimen is:

$$
\boldsymbol{B}_{n e t}=B_{x} \sin (\omega t) \vec{i}+B_{y} \sin (\omega t+\varphi) \vec{j}
$$

where

$\boldsymbol{B}_{n e t}$ is the net magnetic flux density vector. 


\section{$B_{x}, B_{y}$ are the flux density magnitudes generated by the $x$ and $y$ arrays, respectively. \\ $\omega \quad$ is the angular velocity. \\ $\varphi \quad$ is the phase shift angle.}

The angle of the $\boldsymbol{B}_{\text {net }}$ changes continually in a clockwise direction at angular velocity $\omega$. A special case of equal amplitude waveforms with 90 degrees phase shift between them (orthogonal in both space and time), creates a rotating field the test specimen.

It is easy to control the flux density level by controlling the input excitation signals to the array. It is also possible to generate sinusoidal or non-sinusoidal flux densities in the sample according to the input signal shape.

\section{IV.THE NEW TEST FIXTURE STRUCTURE}

This fixture is built of a frame, poles, and sensor coils for measuring the magnetic flux density $(\boldsymbol{B})$ and magnetic field strength $(\boldsymbol{H})$.

\section{A. The magnetic circuit frame}

The frame design is shown in fig. 17 (a). The first component is a $20 \mathrm{~mm}$ thick, flat, circular frame-disc with 16 circular holes drilled around its perimeter. Each hole has a diameter of $51 \mathrm{~mm}$. The frame inner radius is $100 \mathrm{~mm}$, and the outer radius is $170 \mathrm{~mm}$, as shown in fig. 17 (b). The interior space of the frame is a circular indentation with a depth of $10 \mathrm{~mm}$ and a diameter of $200 \mathrm{~mm}$. Its surface functions as a sample holder. This indentation is closed by a moveable cover with the same dimensions and provided with two handles as shown in fig. 17 (a), (b) and (c). The sample will be set between the sample holder and the cover in order to be fixed in the frame center, and in the middle of the frame height. This frame-disc is made from Cast Acrylic [Poly (methyl methacrylate) ], which is a dielectric material ( $\mu_{\mathrm{r}}$ slightly less than 1), that gives the flexibility for the flux to go out of the material to the air and then to the metallic sample. Other properties which encourage use of Acrylic are: its ability to withstand a high temperature (recommended continuous service temperature $85^{\circ} \mathrm{C}$ ), cost effectiveness, superior dimensional stability, durability and ease of assembly [13, 14].

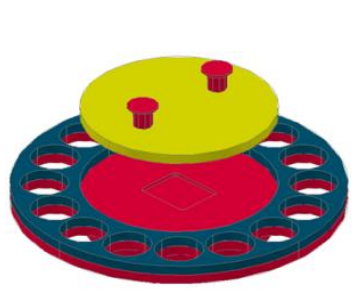

(a)

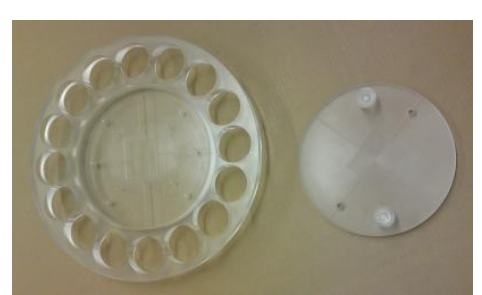

(b)

(c)
Fig. 17: (a) The 3D design of the circuit frame using AutoCAD. (b) The frame disc made of Cast Acrylic. (c) The Cover.

\section{B. The poles}

The assembly consists of 16 poles pairs. One such pole pair is shown in Fig. 18(a). Each circular pole is composed of 56 thin laminations of non-oriented Silicon Steel M19 Gauge 29, and has a diameter of $50 \mathrm{~mm}$. Four slots are laser cut into each lamination to accommodate the windings. Each slot is a square of $10 \mathrm{~mm}$ as shown in fig. 18(b). The interior and exterior corners of the slots are rounded in order to remove any sharp corners in the magnetic circuit. There are two orthogonal windings wrapped around the pole ( $x$-direction coil, and $y$-direction coil). Each layer of the $x$-direction coil is wound so that it is placed on top of a corresponding layer of the $y$-direction coil where the coils intersect at the center of the pole. Thus, the windings are interleaved. The interleaving between the windings increases the homogeneity, uniformity, and similarity between the produced fluxes from each winding.

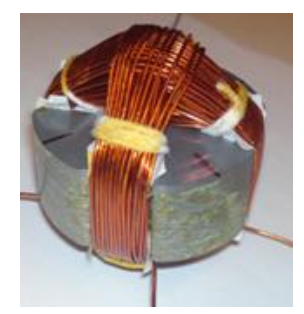

(a)

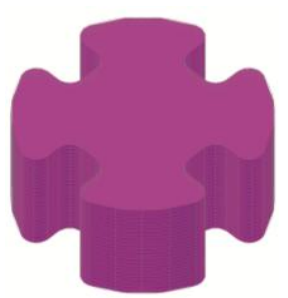

(b)

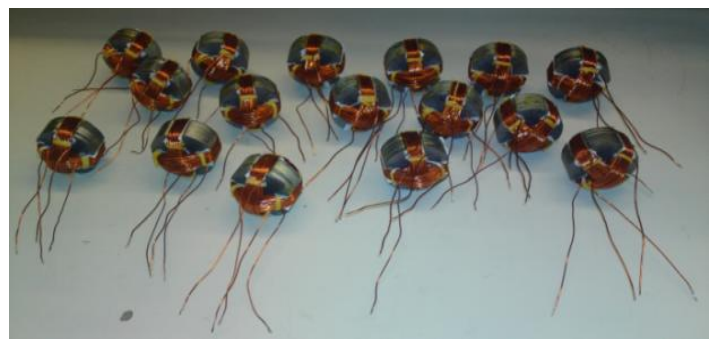

(c)

Fig. 18: (a) Photograph of a pole with wound interleaved coils in $x$ and $y$ directions. (b) Laminations collected to form a pole designed by Auto Cad. (c) A complete set of 16 poles.

\section{The sensor coils for measuring flux density $(\boldsymbol{B})$}

There are at least two methods to measure the flux density in the sample under test. In the needle or tip method, the flux density is calculated by measuring the potential difference between two points on the surface of a steel sheet to which the tips of needles are applied. Its problem appears in the low voltage produced between the needles and susceptibility to the surrounding noise. The other method is sensor coils which are placed within or around the sample [15].

In this design, two sensor coils are employed to measure the magnetic flux density $\boldsymbol{B}$, they are perpendicular to measure $\mathrm{Bx}$ and $\mathrm{By}$, the $x$ and $y$ components, as shown in fig. 19. In prior designs these coils were located in the middle of 
the sample, where holes are drilled and coils wrapped through the holes, to utilize the uniform flux in the middle and to avoid the edges of the sample. But in the current design where the flux is uniform and homogeneous, the coils are wrapped around the outside of the sample. The advantage of the coils being around the sample is allowing the flux to be uniform in the sample. Drilled holes reduce the magnetic quality of the material. The middle area is empty where the tangential $\boldsymbol{H}$ coils can be attached completely to the surface. The $\boldsymbol{B}$ coil width is $180 \mathrm{~mm}$ which is much higher than the typical width $(20 \mathrm{~mm})$; this decreases the chance of coil misalignment between the sensor axis and the field excitation axis, allowing accurate results during $\mathrm{CW}$ and $\mathrm{CCW}$ measurements [16].

The magnetic flux density in the sample can be calculated from Faraday's law, as in equation (5).

$$
B=\left\{\begin{array}{l}
B_{x}=\int \frac{V_{B x}}{N_{B} A_{B}} d t, \text { in } x \text {-direction } \\
B_{y}=\int \frac{V_{B y}}{N_{B} A_{B}} d t, \text { in } y \text {-direction }
\end{array}\right.
$$

Where $\mathrm{V}_{\mathrm{Bx}}$ and $\mathrm{V}_{\mathrm{By}}$ are the terminal voltages of $\boldsymbol{B}$ sensing coils in the $x$ and $y$ directions, respectively. $N_{B}$ is the number of turns around the sample in each direction, and $A_{B}$ is the cross sectional area of the coil.

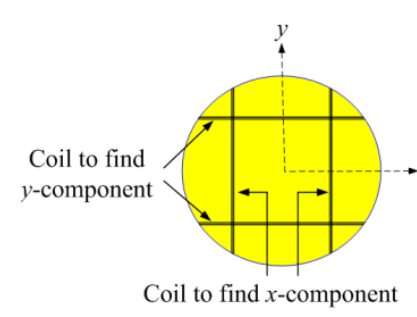

(a)

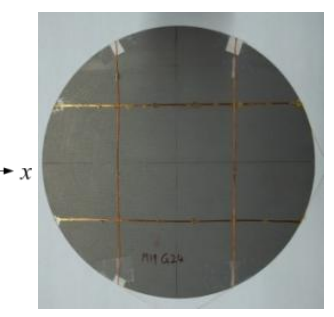

(b)
Fig. 19: The position of $\boldsymbol{B}$ coils wound around the sample. (a) Drawing design. (b) Real picture.

\section{The search coils for measuring magnetic field strength $(\boldsymbol{H})$}

In general, determination of magnetic field strength $\boldsymbol{H}$ at the surface of the electrical steel lamination can be achieved by using the measurement of the current, or the coil method. In the current measurement method, which is also called the indirect method, $\boldsymbol{H}$ is extracted from the measured magnetizing current by applying Ampere's law. This method lacks accuracy, since the magnetic flux path in the circuit is not well defined. It is used widely for pulsating core loss measurement devices, like toroids, Epstein frames, and single sheet testers. Nevertheless, it is not suitable for rotational loss apparatus where the flux path is less distinct [17]. On the other hand, the coil method measures the tangential component of the magnetic field strength at the surface of the sample and can be derived directly from the induced voltage $\mathrm{V}_{\mathrm{H}}$. Different types of sensors may be used to detect tangential magnetic field, such as Rogowski-Chattock potentiometer, tangential coil, Hall sensor, or magnetoresistive sensor [18-201].

In this circuit design, two thin flat multi-turn coils wound on a Plexiglass former are used to measure the tangential components of magnetic field strength $\boldsymbol{H}$. To measure $\boldsymbol{H}$ on the sample surface, the coils are situated perpendicularly in the sample's middle area, one over the sample for sensing Hx, and the other under it for Hy. Figs. 20 (a), (b), and (c) show these coils.

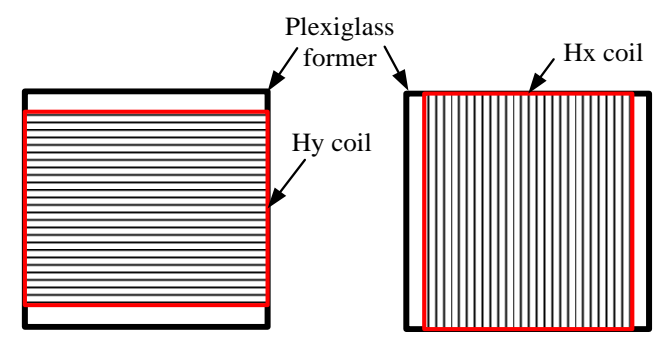

(a)

(b)

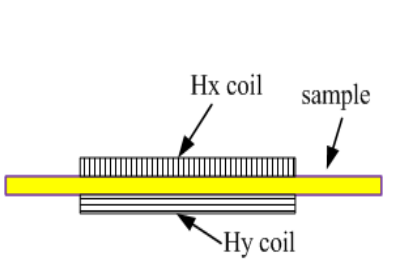

(c)

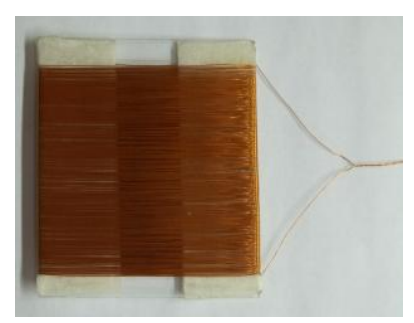

(d)
Fig. 20: (a) $\boldsymbol{H}$ coil for measurement in $y$ direction. (b) $\boldsymbol{H}$ coil for measurement in $x$ direction. (c) Positions of $\boldsymbol{H}$ coil. (d) Real picture of tangential $\boldsymbol{H}$ coil wound on Plexiglass former.

This coil is excited by the magnetic field on the sample surface, and since its former is a non-magnetic material, the magnetic field strength can be calculated by (6).

$$
H=\left\{\begin{array}{l}
H_{x}=\int \frac{V_{H x}}{N_{H} A_{H}} d t, \text { in } x \text {-direction } \\
H_{y}=\int \frac{V_{H y}}{N_{H} A_{H}} d t, \text { in } y \text {-direction }
\end{array}\right.
$$

Where $\mathrm{V}_{\mathrm{Hx}}$ and $\mathrm{V}_{\mathrm{Hy}}$ are the terminal voltages of $\boldsymbol{H}$ sensing coils in the $x$ and $y$ directions, respectively. $N_{H}$ is the number of turns on the former, and $A_{H}$ is the cross sectional area of the coil. Noise affects the output induced voltage from the tangential sensor $\left(\mathrm{V}_{\mathrm{H}}\right)$, since the obtained signal is in the range of a hundred milli volts, thus any small error in the signal appears to be significant in the field strength $\boldsymbol{H}$. To overcome the problem of error, the sensor terminals were twisted as seen in fig. 20 (d), which provides a magnetic field noise reduction. Fig. 21 shows the test fixture of the 16-pole electromagnetic Halbach array. 


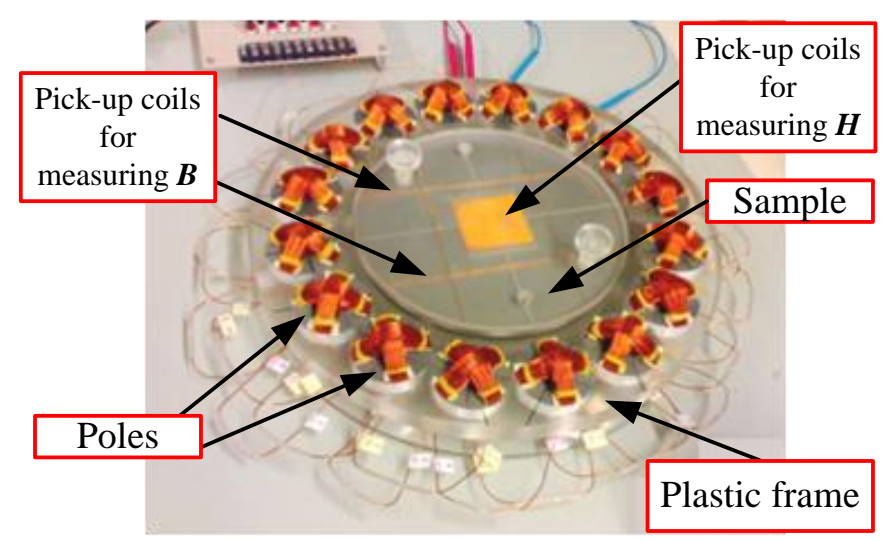

Fig. 21: Photograph of the Halbach Magnetizing Circuit.

\section{V.THE MEASUREMENT SET-UP}

Many methods have been proposed to evaluate the rotational core loss, which can be classified into the following: Torque-metric, thermal, and field-metric methods. Torque-metric method is used in [21], where the difficulty of torque meter construction appears as a major shortcoming. In the thermal method [22], thermocouples are employed to determine the rate of change of the temperature on the sample surface, which is proportional to the dissipated power in the sample: The problem with the thermocouple is its low output voltage signal, and the need for careful installation, calibration, and isolation from noise and electromagnetic interference.

The field-metric method is used in this work, which is seen to be the most convenient and accurate method, plus the ability of providing the $\boldsymbol{B}$ and $\boldsymbol{H}$ waveforms in order to analyze the rotating field behavior along the measurement profile. It is based on the measurements of the magnetic field strength $\boldsymbol{H}$ at the sample surface and flux density $\boldsymbol{B}$ inside the sample [23]. Then, the rotational loss in the lamination can be found from (7).

$$
P=\frac{1}{T \rho} \int_{T}\left(H_{x} \frac{d B_{x}}{d t}+H_{y} \frac{d B_{y}}{d t}\right) d t
$$

Where $H_{x}, H_{y}, B_{x}$, and $B_{y}$ are the measured components of the magnetic field strength and the flux density in $x$ and $y$ directions respectively. $T$ is the time period and $\rho$ is the mass density of the material.

The test bench uses Matlab/Simulink to generate arbitrary waveforms in quadrature to each other; this allows the user flexibility in choosing any physically realizable excitation waveform. These signals are sent through a digital to analogue converter and then isolated by an electronic buffer circuit. Then, two high bandwidth amplifiers receive the signals and amplify them to the required level. The output amplified signals are used to excite the Halbach magnetizing circuit; $\mathrm{Vx}$ excites the $x$-direction array, and $\mathrm{Vy}$ the $y$-direction array. Four output signals from the circuit can be obtained: $\mathrm{V}_{\mathrm{Bx}}, \mathrm{V}_{\mathrm{By}}, \mathrm{V}_{\mathrm{Hx}}$, and $\mathrm{V}_{\mathrm{Hy}}$, which are related to the flux density in the $x$-direction, flux density in the $y$-direction, magnetic field intensity in the $x$-direction, and magnetic field intensity in the $y$-direction, respectively. These signals are sent to dSPACE, which is linked with Matlab Simulink, thus allowing for digital monitoring and control of all generated and received signals. Figs. 22 and 23 illustrate the schematic diagram and the test bench of the measurement system, respectively.

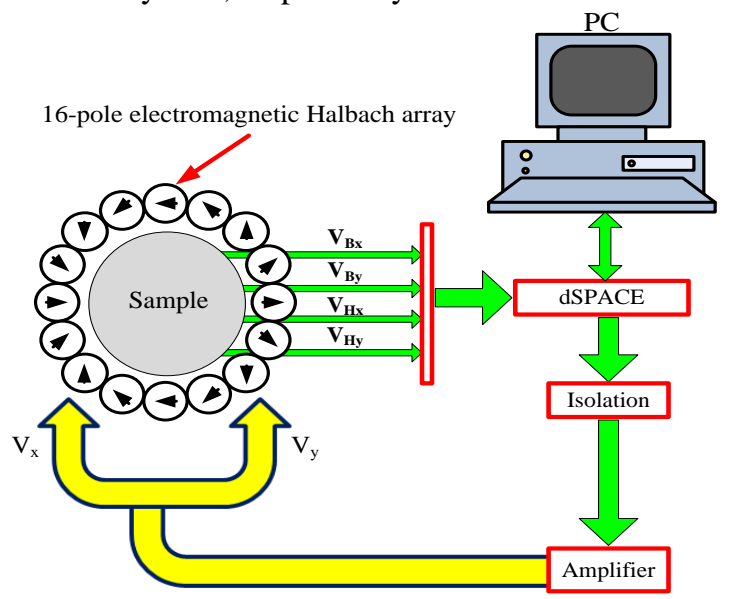

Fig. 22: The test bench schematic diagram.

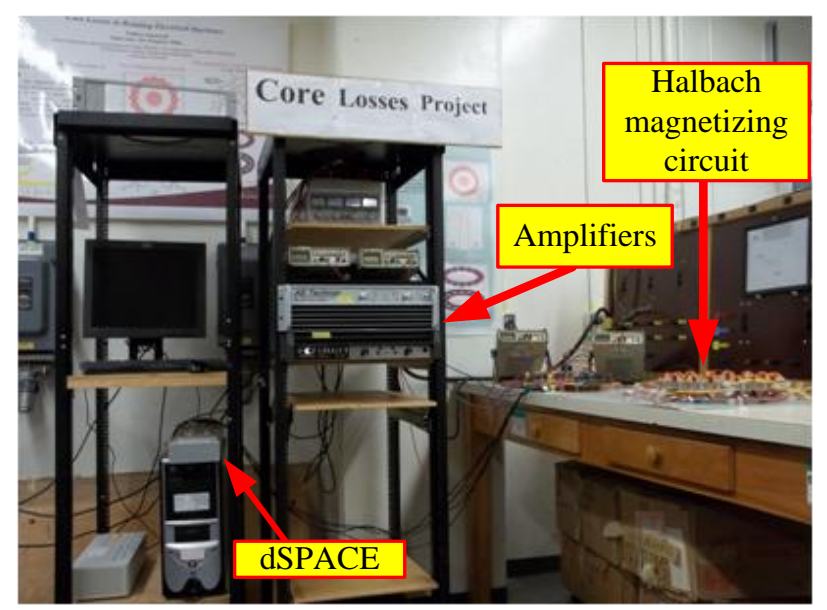

Fig. 23: The measurement system at Concordia University.

\section{EXPERIMENTAL RESULTS OF ROTATIONAL CORE LOSSES}

Both Halbach arrays are exited simultaneously by sinusoidal waveforms at three frequencies of interest to the industry $(60 \mathrm{~Hz}, 400 \mathrm{~Hz}$, and $1 \mathrm{kHz})$, with a phase shift of $90^{\circ}$ between them, where this pattern of fluxes creates a rotating field in the specimen. Two samples of a $20 \mathrm{~cm}$ diameter disc of non-oriented M19 silicon steel gauge 24 and M36 silicon steel gauge 29 are used in this test.

The output voltage signals obtained from the coil sensors during testing of the M36G29 sample are presented at a flux density of $1.3 \mathrm{~T}$, and with frequencies of $60 \mathrm{~Hz}$ and $1 \mathrm{kHz}$. At $60 \mathrm{~Hz}$, the signals obtained from the surface coils $\mathrm{V}_{\mathrm{Hx}}$ 
and $\mathrm{V}_{\mathrm{Hy}}$ are seen in fig. 24, these signals experience some distortion, but the integration reduces the distortion when they are used to construct the magnetic field strength signals $\mathrm{H}_{\mathrm{x}}$ and $\mathrm{H}_{\mathrm{y}}$ as shown in fig. 25. Fig. 26 shows the signals obtained from the $\boldsymbol{B}$ coils, used to evaluate the flux density signals $\mathrm{B}_{\mathrm{x}}$ and $\mathrm{B}_{\mathrm{y}}$ as shown in fig. 27. At $1 \mathrm{kHz}$, the $\mathrm{V}_{\mathrm{Hx}}$ and $\mathrm{V}_{\mathrm{Hy}}$ are seen in fig. 28, which are used to construct the magnetic field strength signals $\mathrm{H}_{\mathrm{x}}$ and $\mathrm{H}_{\mathrm{y}}$ as in fig. 29. Fig. 30 shows $\mathrm{V}_{\mathrm{Bx}}$ and $\mathrm{V}_{\mathrm{By}}$ which are used to construct the flux density signals $\mathrm{B}_{\mathrm{x}}$ and $\mathrm{B}_{\mathrm{y}}$ as shown in fig. 31 .

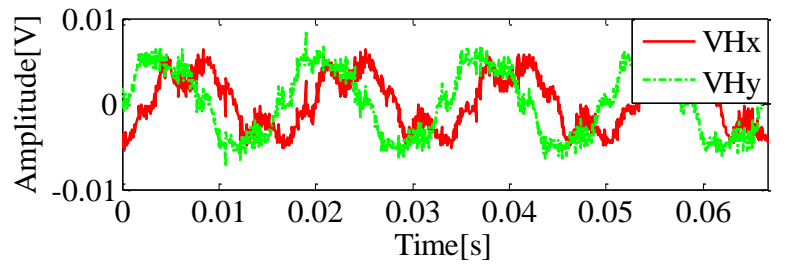

Fig. 24: The instantaneous output voltage signals at $60 \mathrm{~Hz}$ obtained from the surface coils in the $x$-direction $\left(\mathrm{V}_{\mathrm{Hx}}\right)$, and $y$-direction $\left(\mathrm{V}_{\mathrm{Hy}}\right)$.

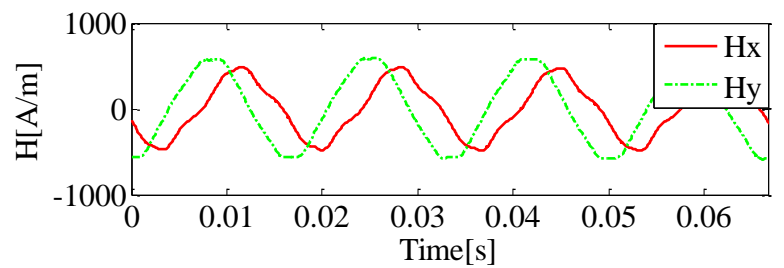

Fig. 25: The instantaneous values of the magnetic field strength at $60 \mathrm{~Hz}$ in the $x$-direction (Hx), and $y$-direction (Hy).

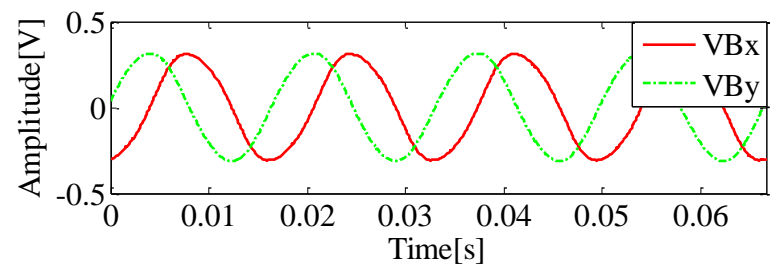

Fig. 26: The instantaneous output voltage signals at $60 \mathrm{~Hz}$ obtained from the $\boldsymbol{B}$ coils in the $x$-direction $\left(\mathrm{V}_{\mathrm{Bx}}\right)$, and $y$-direction $\left(\mathrm{V}_{\mathrm{By}}\right)$.

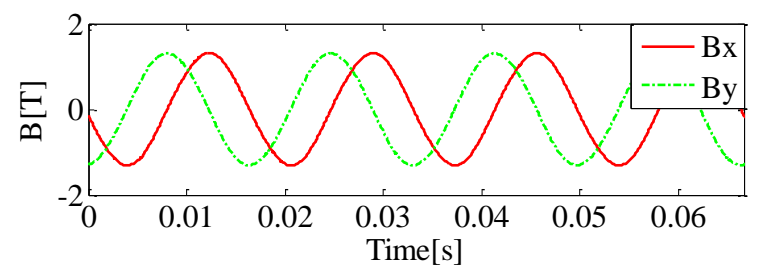

Fig. 27: The instantaneous values of the magnetic flux density at $60 \mathrm{~Hz}$ in the $x$-direction $(\mathrm{Bx})$, and $y$-direction (By).

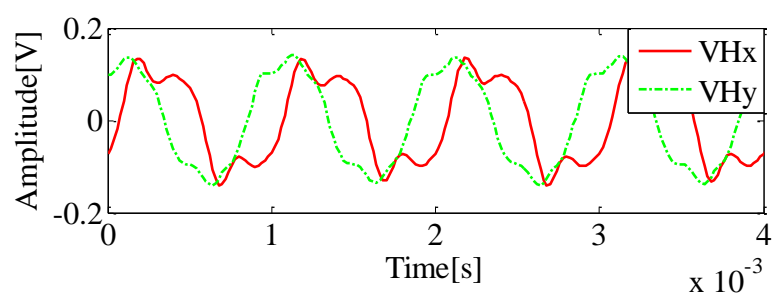

Fig. 28: The instantaneous output voltage signals at $1 \mathrm{kHz}$ obtained from the surface coils in the $x$-direction $\left(\mathrm{V}_{\mathrm{Hx}}\right)$, and $y$-direction $\left(\mathrm{V}_{\mathrm{Hy}}\right)$.

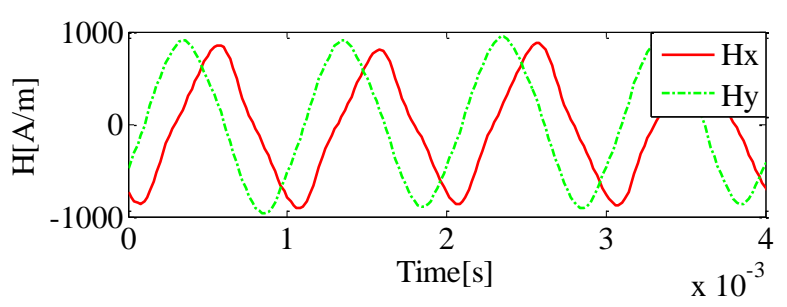

Fig. 29; The instantaneous values of the magnetic field strength at $1 \mathrm{kHz}$ in the $x$-direction (Hx), and $y$-direction (Hy).

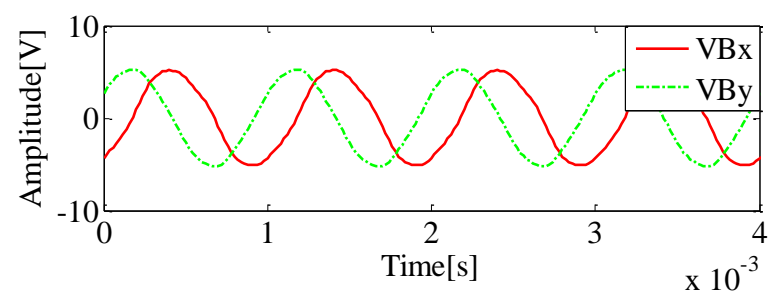

Fig. 30: The instantaneous output voltage signals at $1 \mathrm{kHz}$ obtained from the $\boldsymbol{B}$ coils in the $x$-direction $\left(\mathrm{V}_{\mathrm{Bx}}\right)$, and $y$-direction $\left(\mathrm{V}_{\mathrm{By}}\right)$.

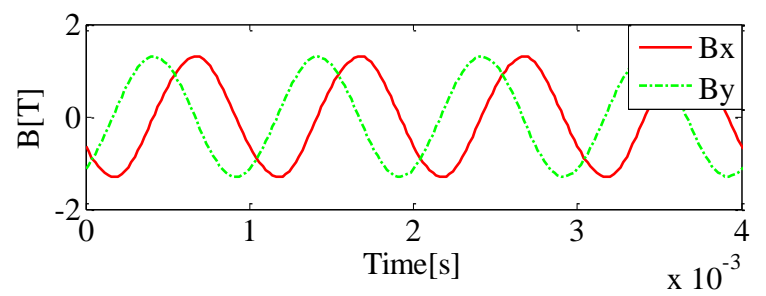

Fig. 31: The instantaneous values of the magnetic flux density at $1 \mathrm{kHz}$ in the $x$-direction $(\mathrm{Bx})$, and $y$-direction (By).

A set of rotational losses $\left(\mathrm{P}_{\mathrm{rot}}\right)$ data was obtained for these two samples with frequencies of $60 \mathrm{~Hz}, 400 \mathrm{~Hz}$, and 1 $\mathrm{kHz}$. In addition, when each one of the Halbach arrays operates alone, a pulsating core loss is produced in the sample, Phx and Phy, the pulsating core losses due to the $x$ and $y$ Halbach arrays, respectively. Results of all frequencies prove that it is not accurate to estimate the rotational core losses as suggested in [24] to avoid the complexity of rotational magnetizing measurements. Superposition gives acceptable estimation for the rotational core losses at low flux density, but as the material goes into saturation, the result becomes over estimated [25]. Fig. 32 shows the results for M19G24 at $60 \mathrm{~Hz}$. The rotational core losses are compared with pulsating losses in the case of 
exciting both $x$ and $y$ arrays separately. In addition, the sum of (Phx and Phy) is benchmarked against these losses. Fig. 33, shows the results for M36G29 at $60 \mathrm{~Hz}$, where in general losses are lower because of the thinner sample of $(0.3556 \mathrm{~mm})$ compared to M19G24 (thickness of $0.635 \mathrm{~mm}$ ). Fig. 34 depicts the difference between rotational core loss and the average of the pulsating core losses, where the rotational core loss is greater than the pulsating loss by almost $45 \%$ on average, and then rotational loss decreases in the saturation region to around $75 \%$ of pulsating loss. There is a clear difference between the rotational and pulsating core losses, not only in the value, but also in the characteristic behavior. The value of $\mathrm{P}_{\text {rot }}$ increases with the applied flux density until a peak value and then decreases, but pulsating loss increases steadily with flux density until the sample is saturated. The reason for that is in the case of rotating field one loss component (let us say $x$-component) increases while the other one ( $y$-component) decreases, and even becomes negative, as seen in fig. 35. This negative power phenomenon has not so far been fully understood by the physicists. In the available literature it is attributed to the silicon iron alloy structure, where the rotation of magnetization lies in an unfavorable direction which results a negative power [26]. This behavior is seen clearly at low frequency and is difficult to achieve at high frequencies, since it is mainly related to the rotational hysteresis loss which is dominant at low frequencies. Figs. 36 and 37 show the loss comparison results for the two samples with a frequency of $400 \mathrm{~Hz}$, and fig. 38 illustrates the difference between the rotational and average pulsating core losses. Figs. 39 and 40 provide the results under a frequency of 1 $\mathrm{kHz}$, and fig. 41 illustrates the difference between rotational and average pulsating core losses, which is around $50 \%$ on average. Here the eddy current loss is more dominant.

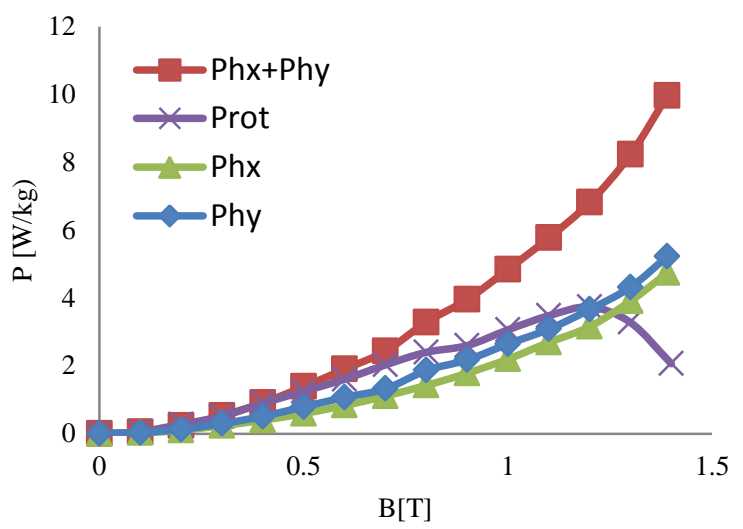

Fig. 32: The rotational core losses $\left(\mathrm{P}_{\mathrm{rot}}\right)$ compared with pulsating losses in $x$ and $y$ directions, Phx and Phy, respectively. For M19G24 at $60 \mathrm{~Hz}$.

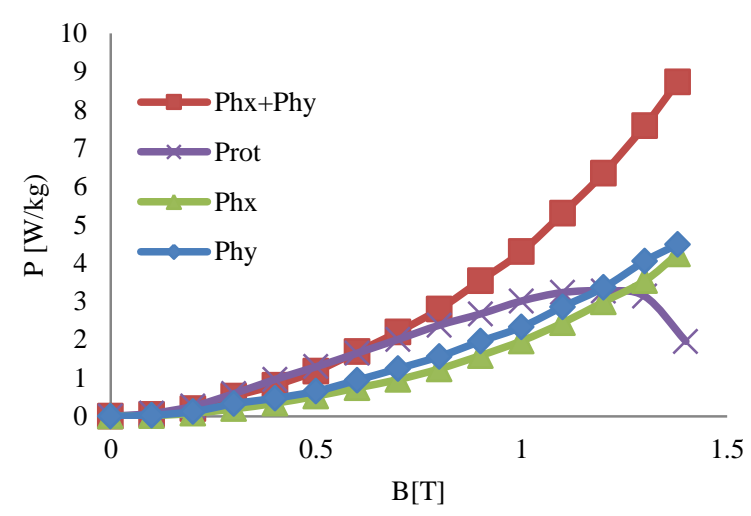

Fig. 33: The rotational core losses $\left(\mathrm{P}_{\text {rot }}\right)$ compared with pulsating losses in $x$ and $y$ directions, Phx and Phy, respectively. For M36G29 at $60 \mathrm{~Hz}$.

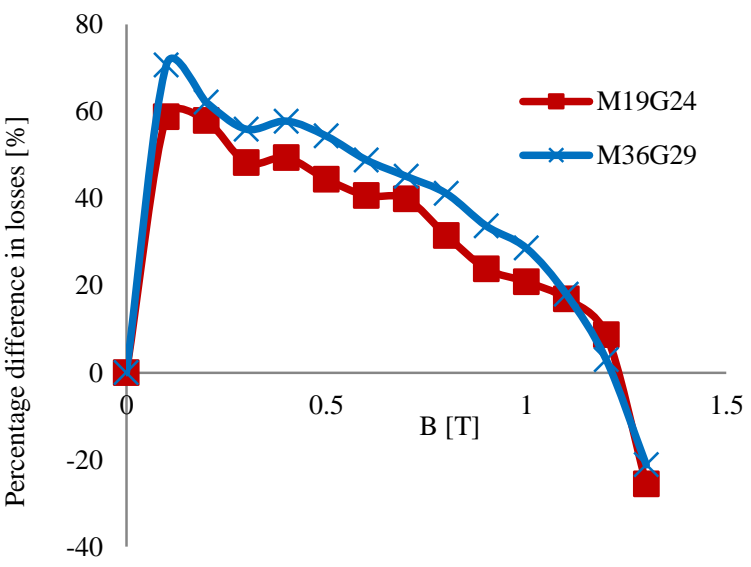

Fig. 34: The difference between rotational core loss and average pulsating core losses, for M19G24 and M36G29 at $60 \mathrm{~Hz}$.

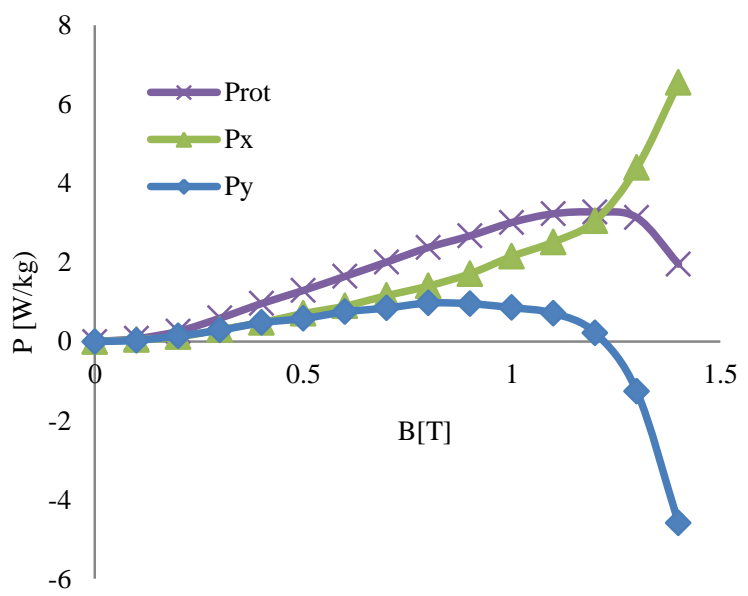

Fig. 35: The total rotational core loss at $60 \mathrm{~Hz}$, in M36 steel Gauge 29, with the corresponding $x$ and $y$ components, Px and Py respectively. 


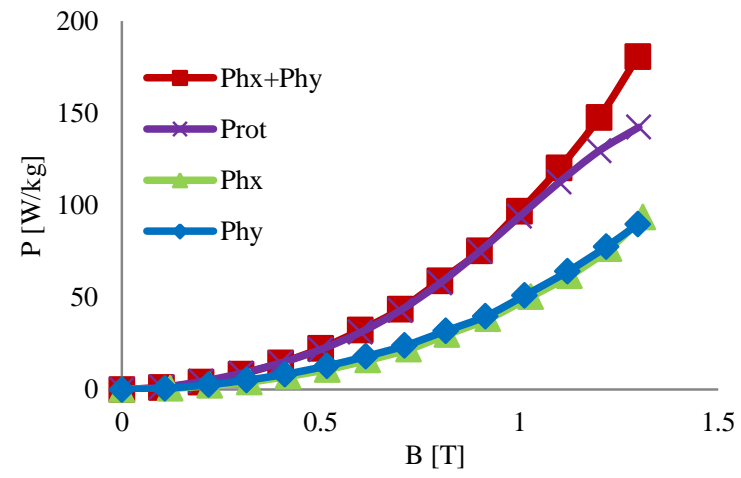

Fig. 36: The rotational core losses $\left(\mathrm{P}_{\text {rot }}\right)$ compared with pulsating losses in $x$ and $y$ directions, Phx and Phy, respectively. For M19G24 at $400 \mathrm{~Hz}$.

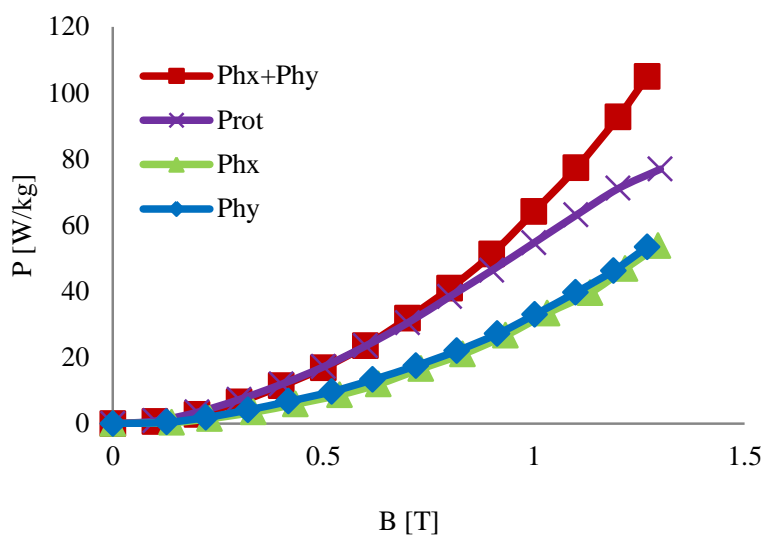

Fig. 37: The rotational core losses $\left(\mathrm{P}_{\text {rot }}\right)$ compared with pulsating losses in $x$ and $y$ directions, Phx and Phy, respectively. For M36G29 at $400 \mathrm{~Hz}$.

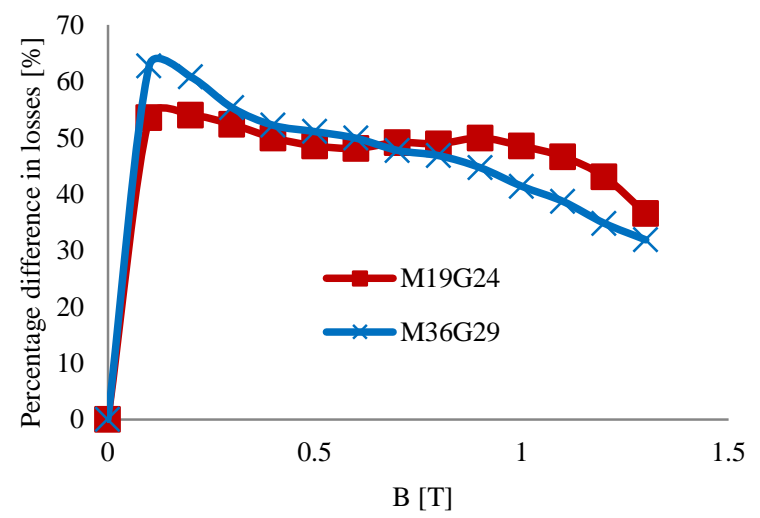

Fig. 38: The difference between rotational core loss and average pulsating core losses, for M19G24 and M136G29 at $400 \mathrm{~Hz}$.

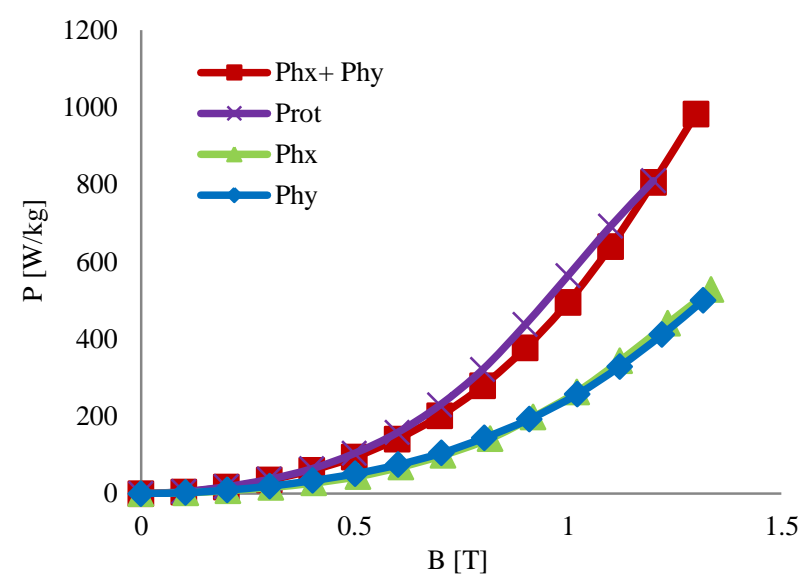

Fig. 39: The rotational core losses $\left(\mathrm{P}_{\text {rot }}\right)$ compared with pulsating losses in $x$ and $y$ directions, Phx and Phy, respectively. For M19G24 at $1 \mathrm{kHz}$.

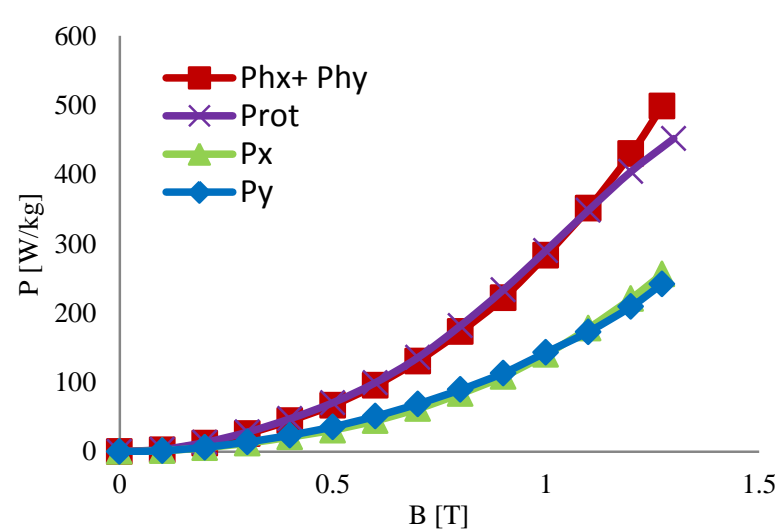

Fig. 40: The rotational core losses $\left(\mathrm{P}_{\text {rot }}\right)$ compared with pulsating losses in $x$ and $y$ directions, Phx and Phy, respectively. For M36G29 at $1 \mathrm{kHz}$.

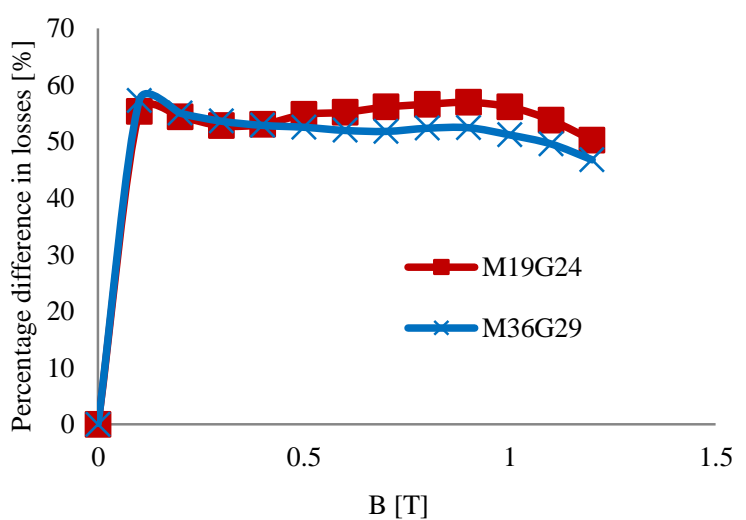

Fig. 41: The difference between rotational core loss and average pulsating core losses, for M19G24 and M136G29 at $1 \mathrm{kHz}$. 


\section{CONCLUSION}

This paper proposes a novel design of an electromagnetic circuit based on the Halbach array principle, which is used for 2-D measurements of rotational core losses in electrical steel laminations. A uniform and homogeneous flux density can be generated within the sample under test, which allows more accurate measurements. In this design, electromagnets are used instead of permanent magnets which leads to a controllable array with compact size. The ability of the electromagnetic Halbach array to generate a uniform and homogeneous flux density distribution within the sample gives the flexibility of using a new technique of wrapping the $\boldsymbol{B}$ coils around the sample, thus allowing the sample to maintain its magnetic proprieties. In addition, the $\boldsymbol{B}$ coils are wrapped into two blocks to leave the sample middle area empty, which gives the opportunity for the $\boldsymbol{H}$ coils to be placed directly on the sample. Thus a higher accuracy for magnetic field intensity measurements on the sample surface can be obtained. The $\boldsymbol{B}$ coil width is much higher $(180 \mathrm{~mm})$ compared with the typical coil width $(20 \mathrm{~mm})$ which decreases the possibility of misalignments in $\boldsymbol{B}$ measurements in the clockwise and counterclockwise directions.

The circuit is simulated, prototyped, and tested. The rotational and pulsating core losses are measured for two circular samples of non-oriented silicon steel M19 gauge 24, and M36 gauge 29, at three frequencies of industrial interest $(60 \mathrm{~Hz}, 400 \mathrm{~Hz}$, and $1 \mathrm{kHz})$. The field-metric method is used in this work, which is seen to be the most convenient and accurate one compared to other methods.

Future work and research trends are concentrated towards studying many physical phenomena associated with the measurements of rotational core losses, such as the difference in loss in $\mathrm{CW}$ and $\mathrm{CCW}$ directions, the negative power which has appeared at one loss component measurement, and the effect of harmonics and its relation with the B-H loop trajectory.

\section{ACKNOWLEGMENT}

This project is part of the R\&D program of the NSERC Chair entitled "Energy efficiency in electrical machines for small renewable energy production systems" established in 2009 at Concordia University. The authors acknowledge the support of the Natural Sciences \& Engineering Research Council of Canada and Hydro-Québec.

\section{REFERENCES}

[1] A. J. Moses, "Importance of rotational losses in rotating machines and transformers," J. Mater. Eng. Perform. (JMEPEG), vol. 12, pp. 235-244, 1992.

[2] G. Diaz, et al., "Analytical Interpretation and Quantification of Rotational Losses in Stator Cores of Induction Motors," IEEE Transactions on Magnetics, vol. 43, pp. 3861-3867, 2007.

[3] Y. Guo, J. G. Zhu, J. Zhong, H. Lu, and J. X. Jin, "Measurement and modeling of rotational core losses of soft magnetic materials used in electrical machines, A Review," IEEE Trans. on Magn, vol. 44, pp. 279-291,2008.

[4] J. G. Zhu and V. S. Ramsden, "Improved formulations for rotational core losses in rotating electrical machines," IEEE Trans. on Magn, vol. 34, pp. 2234-2242, 1998

[5] N. Alatawneh and P. Pillay, "Test Specimen Shape Considerations for the Measurement of Rotational Core Losses," IEEE Transactions on Energy Conversion, vol. 27, pp. 151-159, 2012.

[6] J. Sievert, et al., "European intercomparison of measurements of rotational power loss in electrical sheet steel," Journal of Magnetism and Magnetic Materials, vol. 160, pp. 115-118, 1996.

[7] G. Krismanic, "Recent developments and trends in measurements of two-dimensional magnetic properties," Journal of Electrical Engineering, vol. 55, pp. 45-48, 2004.

[8] J. C. Mallinson, "One-sided fluxes-A magnetic curiosity?" IEEE Trans .on Magn., vol. MAG-9, pp. 678-682, Dec. 1973.

[9] Jae-Seok Choiand, Jeonghoon Yoo, "Design of a Halbach Magnet Array Based on Optimization Techniques," IEEE Trans. on Magn., vol. 44, pp. 2366, Oct. 2008.

[10] H. Raich, P. Blümler, "Design and construction of a dipolar Halbach array with a homogeneous field from identical bar magnets: NMR Mandhalas," Concepts in Magnetic Resonance Part B-Magnetic Resonance Engineering, vol. 23B, pp. 16-25, 2004.

[11] Klaus Halbach; "Physical and Optical Properties of Rare Earth Cobalt Magnets," Nuclear Instruments and Methods, 187, pp. 109-117, (1981).

[12] Z. Q. Zhu and D. Howe, "Halbach permanent magnet machines and applications: a review," IEE Proceedings-Electric Power Applications, vol. 148, pp. 299-308, 2001.

[13] Ashby, Michael F. (2005). Materials Selection in Mechanical Design (3rd ed.). Elsevier. ISBN 0-7506-6168-2, p 519.

[14] Physical Properties of Acrylic Sheets, Data sheet, http://www.kaysons.in.

[15] N. Stranges, "An investigation of iron losses due to rotating flux in three phase induction motor cores", Ph.D.thesis, McMaster University, Canada, Dec. 2000.

[16] S. Zurek and T. Meydan, "Errors in the power loss measured in clockwise and anticlockwise rotational magnetisation. Part 2: Physical phenomena," IEE Proceedings Science, Measurement and Technology, vol. 153, pp. 152-157, 2006.

[17] H. Pfutzner and P. Schonhuber, "On the problem of the field detection for single sheet tests," IEEE Trans. on Magn, MAG 27, p. $778,1991$.

[18] S. Tumanski, "Induction coil sensors a review," Meas. Sci. Technol., no. 3, pp. R31-R46, 2007.

[19] S. Tumanski, "A multi-coil sensor for tangential magnetic field investigations," J. Magn. Magn. Mater. 242-245 (2002) 1153-1156.

[20] H. Zijlstra, "Experimental methods in magnetism", vol.2, North Holland Publishing Company- Amsterdam, p.p 56-59, 1967.

[21] F. Brailsford, "Rotational hysteresis loss in electrical sheet steels," Journal of the Institution of Electrical Engineers, vol. 83, pp. 566-575, 1938.

[22] C. R. Boon and J. E. Thompson, "Alternating and rotational power loss at $50 \mathrm{c} / \mathrm{s}$ in $3 \%$ silicon-iron sheets," Proceedings of the Institution of Electrical Engineers, vol. 112, pp. 2147-2151, 1965.

[23] K. Atallah and D. Howe, "Calculation of the rotational power loss in electrical steel laminations from measured $\mathrm{H}$ and B," IEEE Trans. on Magn, vol. 29, no. 6, pp. 3547-3549, 1993.

[24] A. J. Moses, "Rotational magnetization-problems in experimental and theoretical studies of electrical steels and amorphous magnetic materials," IEEE Trans. on Magn, vol. 30, pp. 902-906, 1994.

[25] N. Stranges and R. D. Findlay, "Measurement of rotational iron losses in electrical sheet," IEEE Trans. on Magn, vol. 36, pp. $3457-3459,2000$.

[26] J. J. Zhong, J. G. Zhu, Y. G. Guo, and Z. W. Lin, "Improved measurement with 2-D rotating fluxes considering the effect of internal field, "IEEE Transactions on Magnetics", vol. 41, pp. 3709-3711, 2005. 


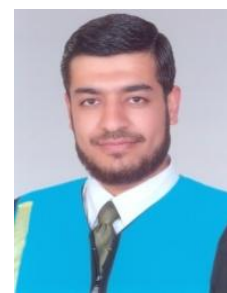

Natheer Alatawneh (S'09) was born in Amman, Jordan. He received the M.S. degree in Electrical Engineering from Jordan University of Science and Technology, Irdid, Jordan, in 2004. He is currently working toward the Ph.D. degree at Concordia University, Montreal, Canada.

His research interests include core losses in AC machines, modeling and analysis of electric machines, and machine drives.

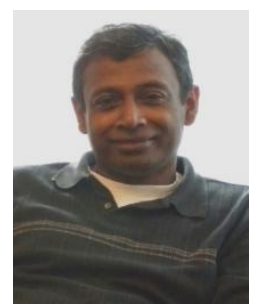

Pragasen Pillay ( F'05) received the Bachelor's degree from the University of Durban-Westville, Durban, South Africa, in 1981, the Master's degree from the University of Natal, Durban, South Africa, in 1983, and the Ph.D. degree from Virginia Polytechnic Institute and State University, Blacksburg, in 1987.

Currently, he is a Professor in the Department of Electrical and Computer Engineering, Concordia University, Montreal, Quebec, where he is the Senior Hydro Quebec Chair. From 1988 to 1990, he was with the University of Newcastle-upon-Tyne, Newcastle-upon-Tyne, U.K. From 1990 to 1995, he was with the University of New Orleans. From 1995-2007 he was at Clarkson University, Potsdam, NY, where he held the Jean Newell Distinguished Professorship in Engineering. He is also an Adjunct Professor at the University of Cape Town, Cape Town, South Africa. His research and teaching interests are in modeling, design, and control of electric motors and drives for industrial and alternate energy applications.

Dr. Pillay is a member of the IEEE Power Engineering, IEEE Industry Applications (IAS), IEEE Industrial Electronics, and IEEE Power Electronics Societies. He is a member of the Electric Machines Committee and Past Chairman of the Industrial Drives Committee of the IAS and Past Chairman of the Induction Machinery Subcommittee of the IEEE Power Engineering Society. He currently chairs the Awards Committee of the IAS Industrial Power Conversion Department. He has organized and taught short courses in electric drives at IAS Annual Meetings. He is a Fellow of the Institution of Electrical Engineers, U.K., and a Chartered Electrical Engineer in the U.K. He is also a Member of the Academy of Science of South Africa. He was also a recipient of a Fulbright Scholarship. 